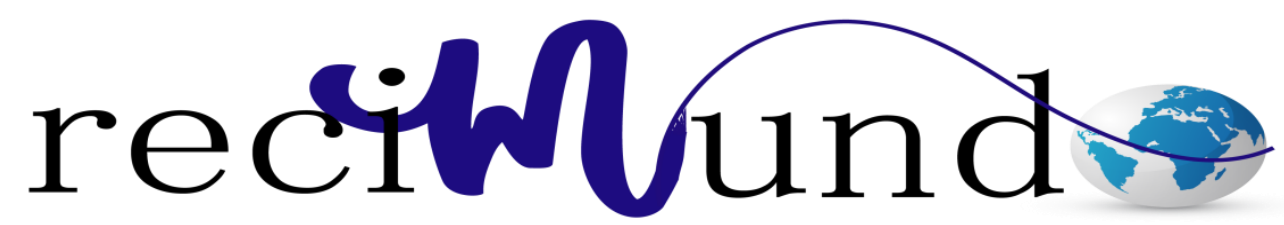

Revista Científica Mundo de la Investigación y el Conocimiento

Miguel Augusto Baque Cantos ${ }^{\text {a }}$, Luz Teresa Cañarte Quimis ${ }^{\text {b }}$; José Luis Merino Murillo $^{c}$; Mariana de Lourdes Cantos Figueroa ${ }^{\mathrm{d}}$

Análisis de la cultura organizacional en los departamentos de talento humano y financiero del G.A.D de Jipijapa

Revista Científica Mundo de la Investigación y el Conocimiento. Vol. 1 núm., 5, diciembre, 2017, pp. 944-959

DOI: 10.26820/recimundo/1.5.2017.944-959

Editorial Saberes del Conocimiento

a. Ingeniero Comercial de la Universidad Técnica de Manabí; Diplomado en Autoevaluación y Acreditación Universitaria de la Universidad Aconcagua República de Chile; Magister en Docencia Universitaria e Investigación Educativa de la Universidad Nacional de Loja; Docente principal titular Universidad Estatal del Sur de Manabí; miguel.baque@ hotmail.com - miguel.baque@unesum.edu.ec.

b. Ingeniera Comercial de la Universidad Técnica de Manabí; Master en Docencia Mención: Gestión Desarrollo del Currículo; Diplomado "Autoevaluación y Acreditación Universitaria" de la Universidad Aconcagua, Republica de Chile; Docente Principal Titular de la Universidad Estatal del Sur de Manabí; luztere2006@hotmail.com-luz.canarte@unesum.edu.ec.

c. Ingeniero Comercial de la Universidad Laica Eloy Alfaro de Manabí; Magister en Finanzas y Comercio Internacional de la Universidad Laica Eloy Alfaro de Manabí; Docente: Contratado: Tiempo completo de la Carrera Comercio Exterior de la UNESUM; joselu_merino28@ hotmail.com -jose.merino@unesum.edu.ec.

d. Ingeniera Comercial de la Universidad Técnica de Manabí; Diplomado en Autoevaluación y Acreditación Universitaria de la Universidad Aconcagua de la República de Chile; Magister en Docencia Universitaria e Investigación Educativa Universidad Nacional de Loja; Docente Principal Titular de la Universidad Estatal del Sur de Manabí; macafi1@yahoo.com _mariana.cantos@unesum.edu.ec. 


\section{Análisis de la cultura organizacional en los departamentos de talento humano y financiero del G.A.D de Jipijapa Vol. 1, núm. 5., (2017)}

Miguel Augusto Baque Cantos; Luz Teresa Cañarte Quimis; José Luis Merino Murillo; Mariana de Lourdes Cantos Figuero

\section{RESUMEN}

El estar en un entorno organizado y planificado es lo mejor que se puede tener dentro de una organización y más si esta es pública, ya que los procesos administrativos y de control interno en Latinoamérica dejan mucho que desear, la corrupción y burocracia en algunos casos está a la orden del día. Generalmente estos comportamientos están asociados a una falta de formación al talento humano dentro de nuestras estructuras de administración pública y más aún cuando estos están descentralizados y tienen que generar sus propios recursos para ser auto gestión, el correcto manejo de los recursos es fundamental y a ello no escapa G.A.D de jipijapa. La metodología de investigación utilizada fue de tipo revisión bibliográfica referenciada en páginas web, artículos de opinión, revistas científicas, sobre talento humano, administración de recursos y cultura organizacional en este caso asociados al Cantón de Jipijapa. Entre los resultados obtenidos es que se realizó un diagnóstico sobre los procesos administrativa del GAD Municipal del cantón Jipijapa y se concluyó que el Departamento Administrativo no cuenta con la ejecución de una serie de funciones en los procesos y capacitación del personal, que brinde facilidades para una buena administración y dentro de las conclusiones el Cantón JIPIJAPA no dispone de Políticas y Procedimientos para la contratación del personal.

Palabras clave: Talento, humano, administración, financiero, contratación. 
Análisis de la cultura organizacional en los departamentos de talento humano y financiero del G.A.D de Jipijapa

Vol. 1, núm. 5., (2017)

Miguel Augusto Baque Cantos; Luz Teresa Cañarte Quimis; José Luis Merino Murillo; Mariana de Lourdes Cantos Figuero

\section{ABSTRACT}

Being in an organized and planned environment is the best that can be had within an organization and more if it is public, since the administrative processes and internal control in Latin America leave much to be desired, corruption and bureaucracy in some cases is To the order of the day. Generally, these behaviors are associated with a lack of training for human talent within our public administration structures and even more so when these are decentralized and have to generate their own resources to be self-managed, the correct management of resources is fundamental and it is not Gad escapes from jipijapa. The research methodology used was of bibliographic review type referenced in web pages, opinion articles, scientific journals, on human talent, resource management and organizational culture in this case associated with the Canton of Jipijapa. Among the results obtained is that a diagnosis was made of the administrative processes of the Municipal GAD of the canton Jipijapa and it was concluded that the Administrative Department does not have the execution of a series of functions in the processes and personnel training, which provides facilities for a good administration and within the conclusions the Canton JIPIJAPA does not have Policies and Procedures for the hiring of personnel.

Keywords: Talent, human, administration, financial, hiring. 


\section{Análisis de la cultura organizacional en los departamentos de talento humano}

y financiero del G.A.D de Jipijapa

Vol. 1, núm. 5., (2017)

Miguel Augusto Baque Cantos; Luz Teresa Cañarte Quimis; José Luis Merino Murillo; Mariana de Lourdes Cantos Figuero

\section{Introducción.}

Los procesos administrativos dentro de las instituciones públicas o privadas, como es el caso de los Gobiernos Autónomos Descentralizados "GAD's”, que es el efecto y la acción de realizar gestiones y de administrar la eficiencia de la institución. Desde este punto de vista se generaliza el proceso administrativo que es la acción de planificar, organizar, dirigir y controlar el desarrollo de las actividades dentro de los Municipios o GAD`s, compaginados con todos los demás recursos organizacionales, con el objetivo o propósito de lograr las metas establecida en la institución (Villacreses, 2017).

La gestión de talento humano ha evolucionado radicalmente dentro de las organizaciones debido a que el personal o capital humano es la parte fundamental para el crecimiento y desarrollo de las instituciones, es por todo esto que hoy en día se ha visto la necesidad de contar con un Modelo de Gestión de Talento Humano que le permita a la persona encargada de talento humano contar con una herramienta importante la misma que le permita llevar adelante los procesos que engloba el desarrollo y mejoramiento de las personas a nivel institucional (Vallejos \& Robinzon, 2017).

Actualmente en esta época globalizada el área de talento humano en toda entidad, tanto pública como privada está expuesto a cometer errores lo cual demanda de un control rígido de todos los procesos y políticas que asume una entidad determinada dentro de un tiempo definido y es aquí donde la auditoría de gestión actúa como una herramienta de control que permite evaluar cada una de los procesos realizados dentro del área (Sánchez \& Lucio, 2017). La idea fundamental es que por medio de los procesos de auditorías se determinen los errores si los 
Análisis de la cultura organizacional en los departamentos de talento humano y financiero del G.A.D de Jipijapa

Vol. 1, núm. 5., (2017)

Miguel Augusto Baque Cantos; Luz Teresa Cañarte Quimis; José Luis Merino Murillo; Mariana de Lourdes Cantos Figuero

hubiere, para de esta manera hacer los planes de acción y corregirlos, lo que permitirá tener una organización con trabajadores altamente capacitados y sin problemas organizacionales.

Existen principios fundamentales que norman, regulan, amparan y protegen el desarrollo el trabajador en el desempeño de sus funciones, propiciando su capacitación, el respeto a sus derechos, el cumplimiento de sus obligaciones en términos de eficacia, eficiencia y efectividad además de la selección del personal adecuado, la formación específica del mismo, el adiestramiento, la evaluación, la remuneración justa y la promoción serán los pilares básicos para la conformación de una institución con visión de futuro y dispuesta a enfrentar los desafíos del nuevo milenio (Sánchez \& Lucio, 2017). El pilar fundamental de toda organización es su talento humano, mientras más se sienta el trabajador comprometido con su trabajo y su empresa, mayores serán los niveles de productividad. Y a su vez la empresa debe hacer todo lo necesario para estimular y reconocer la capacidad que tienen los trabajadores de hacer andar la organización.

(Chiavenato, 2009) sostiene que la formación mejora la productividad en la empresa, asegura la calidad del trabajo del personal, motiva al personal en sus puestos de trabajo permitiendo el cumplimiento de objetivos planeados brinda seguridad interna entre compañeros de trabajo puesto que hay un comunicación afectiva en las empresas (Vallejos \& Robinzon, 2017). Es una inversión necesaria que debe hacer la empresa para mantener los niveles de armonía dentro de su talento humano, inversión que se verá retribuida en el mediano plazo, en base a la productividad y el empeño del trabajar día a día para hacer de su empresa exitosa. 


\section{Análisis de la cultura organizacional en los departamentos de talento humano}

y financiero del G.A.D de Jipijapa

Vol. 1, núm. 5., (2017)

Miguel Augusto Baque Cantos; Luz Teresa Cañarte Quimis; José Luis Merino Murillo; Mariana de Lourdes Cantos Figuero

En la actualidad las instituciones del Estado se encuentran en un proceso de transición que tiene por objetivo brindar a los ciudadanos servicios de calidad, dejando atrás la anterior imagen del servidor público ineficiente y carente de empatía por el trabajo que desempeña. La gestión del talento humano se encarga de obtener y coordinar a las personas de una organización, de forma que consigan las metas establecidas (Fernández, 2017).

En la actualidad la gestión del talento humano tiene que ser más subjetiva y más afinada: determinando los requerimientos de los recursos humanos, acrecentando las fuentes más efectivas que permitan allegarse a los candidatos idóneos, evaluando la potencialidad física y mental de los solicitantes, así como su aptitud para el trabajo, utilizando para ello una serie de técnicas, como la entrevista, las pruebas psicometrías y los exámenes médicos (Fernández, 2017). Es decir lo métodos de selección de aspirantes a trabajar dentro de las instituciones del Estado, deben ser rigurosas, en muchos casos las personas buscan trabajarle al estado para convertirse en funcionarios públicos mas no servidores públicos, en muchos casos para obtener prebendas personales o aprovecharse del cargo y esto genera niveles de burocracia y corrupción que afectan la imagen de la institución pública.

Las instituciones públicas tienen la responsabilidad de cumplir con los procesos presupuestarios de acuerdo a cada una de las obligaciones ya que ahí radica la calidad de gasto y la responsabilidad de los controles interno para la seguridad financiera y contable de la institución (Álvarez \& Guachi, Análisis del Sistema de Control Interno y su Impacto en el Proceso de Ejecución Presupuestaria del Gobierno Autónomo Descentralizado del Cantón Jipijapa, 2017). Por ello también las instituciones públicas deben cotejar el gasto público con sus 
Análisis de la cultura organizacional en los departamentos de talento humano y financiero del G.A.D de Jipijapa

Vol. 1, núm. 5., (2017)

Miguel Augusto Baque Cantos; Luz Teresa Cañarte Quimis; José Luis Merino Murillo; Mariana de Lourdes Cantos Figuero

presentaciones anuales de rendición de cuentas que en otros países es llamado memorias y cuentas. Esto hace que se pueda medir lo planificado dentro de todos los planes y proyectos que se quieren realizar con lo que se ha ejecutado, a su vez que estas herramientas son importantes como indicador de gestión y da elementos importantes de cómo va encaminada la gestión de gobierno.

El control interno juega un papel preponderante en el gasto público, y su cumplimiento de acuerdo a los requerimientos que exigen las entidades de control del estado como la Contraloría, la Superintendencia de Banco y otras entidades de control (Álvarez \& Guachi, Análisis del Sistema de Control Interno y su Impacto en el Proceso de Ejecución Presupuestaria del Gobierno Autónomo Descentralizado del Cantón Jipijapa, 2017). Los procesos de auditoria lo que buscan es eso, que lo presupuestado se haya ejecutado y de no haberse ejecutado, buscar la respuestas del porque no hubo ejecución, el inadecuado uso del dinero publico genera responsabilidades penales y administrativas.

La gestión administrativa en la localidad de Jipijapa es uno de los factores más importantes cuando se trata del desarrollo de la misma y para lograrlo es necesario contar con el primer ente que integra la administración local como es el Gobierno autónomo descentralizado municipal del Cantón Jipijapa, siendo esta una institución del gobierno en la que tiene por objetivo proveer de servicios a los habitantes en diversos niveles jerárquico ya que a través de procesos específicos y una adecuada coordinación permitirá el uso eficiente de los recursos económicos, materiales, humanos logrando así el éxito de cumplir con los requerimientos de los ciudadanos (Álvarez \& Vera, La Gestión Administrativa y de Control y su incidencia en el Área 


\section{Análisis de la cultura organizacional en los departamentos de talento humano}

y financiero del G.A.D de Jipijapa

Vol. 1, núm. 5., (2017)

Miguel Augusto Baque Cantos; Luz Teresa Cañarte Quimis; José Luis Merino Murillo; Mariana de Lourdes Cantos Figuero

de Gestión de Planificación y del territorio del Gobierno Autónomo descentralizado Municipal del Cantón Jipijapa, 2017).

\section{Materiales y métodos.}

La investigación bibliográfica es la primera etapa del proceso investigativo que proporciona el conocimiento de las investigaciones ya existentes, de un modo sistemático, a través de una amplia búsqueda de: información, conocimientos y técnicas sobre una cuestión determinada (Mora, 2018)

El proceso de investigación estará completo cuando se cumpla el objetivo de la investigación científica: un documento científico al cual los siguientes usuarios buscarán como referencia, de tal manera que observarán hechos, plantearán problemas; funcionando así, como un nuevo punto de partida, realizado con la mayor objetividad posible, para futuras investigaciones (Mora, 2018).

La metodóloga empleada para esta investigación es documental de tipo bibliográfica, en vista que los elementos que se utilizaron como referencias para la realización de este articulo fueron medios electrónicos, como páginas web, artículos de revistas, entre otros, donde había información importante del objeto de estudio de esta investigación.

Jipijapa está ubicado al sur de Manabí y es conocido también como "La Sultana del Café", por ser el principal productor de café en el Ecuador. Su nombre se debe a que antiguamente estuvo poblada por la tribu indígena Xipixapa, aunque se especula que también los Mayas se extendieron por esta región. La principal actividad de este cantón es la producción y 
Análisis de la cultura organizacional en los departamentos de talento humano y financiero del G.A.D de Jipijapa

Vol. 1, núm. 5., (2017)

Miguel Augusto Baque Cantos; Luz Teresa Cañarte Quimis; José Luis Merino Murillo; Mariana de Lourdes Cantos Figuero

exportación de café, además del comercio en sus diferentes formas y la artesanía (Gobierno Autonomo Descentralizado del Cantón Jipijapa, 2018).

\section{Resultados.}

- Se realizó un diagnóstico sobre los procesos administrativa del GAD Municipal del cantón Jipijapa y se concluyó que el Departamento Administrativo no cuenta con la ejecución de una serie de funciones en los procesos y capacitación del personal, que brinde facilidades para una buena administración, gran parte de las personas encuestadas en un $81 \%$ conocen de los objetivos que están planteados en la institución, pero se nota la gran debilidad en su proceso de ejecución. Otro aspecto importante que se pudo detectar en la encuesta que el 78\% indicó que no se está cumpliendo con la administración por proceso, donde se encuentran con incongruencias al momento de ser detallado al inicio del periodo, por la falta de información de los y las servidoras públicas, ya que no se prioriza un análisis a la situación interna y externa del G.A.D. De esta manera queda una falencia en cuanto a la planificación, viendo afectado el progreso de la entidad (Villacreses, 2017).

- Los resultados de la encuesta en un 52\% manifestaron que no se ha logrado un desarrollo institucional, considerando que el grado de incidencia es medio con un $43 \%$, es decir, desde una perspectiva funcionalista se afirma que la función básica de la administración, como práctica, es la de seleccionar objetivos apropiados y dirigir a la organización hacia su logro. De igual manera se verifico que el grado de desempeño de las actividades administrativas fueron calificado de bueno, lo que demuestra que estas no 


\section{Análisis de la cultura organizacional en los departamentos de talento humano}

y financiero del G.A.D de Jipijapa

Vol. 1, núm. 5., (2017)

Miguel Augusto Baque Cantos; Luz Teresa Cañarte Quimis; José Luis Merino Murillo; Mariana de Lourdes Cantos Figuero

satisfacen las necesidades para el cumplimiento de los objetivos propuestos, por lo tanto es necesario que se logre cumplir para activar así el desarrollo institucional del GAD (Villacreses, 2017).

- Los procesos administrativos en el Gobierno Autónomo Descentralizado Municipal del cantón Jipijapa, presentan una débil articulación al momento de evaluar el desempeño del departamento administrativo de la institución, esta deficiencia que se presenta en el GAD Municipal del cantón Jipijapa, se da por cuanto la institución dispone de un Modelo de procesos administrativos pero mal ejecutado que guíe una estructura a seguir. Luego de conocer los procesos se evidenció que la realización de funciones por parte de los y las servidoras públicas no son controladas y esto ocasiona un incumplimiento en la asignación de funciones básicas de planeación, organización, dirección y control y no contribuye eficazmente en los principios de dirección en el GAD (Villacreses, 2017).

\section{Conclusiones.}

- El proceso de reclutamiento, selección del personal, no se realiza mediante concurso de méritos y oposición como lo dispone la LOSEP y la Constitución del Ecuador la aplicación de este método brinda confianza y cumple con las normas y políticas establecidas (Sánchez \& Lucio, 2017).

- El GAD del Cantón JIPIJAPA no dispone de Políticas y Procedimientos para la contratación del personal, debido que no existe una ordenanza donde especifique cumplir con las Políticas de contratación (Sánchez \& Lucio, 2017) 
Análisis de la cultura organizacional en los departamentos de talento humano y financiero del G.A.D de Jipijapa

Vol. 1, núm. 5., (2017)

Miguel Augusto Baque Cantos; Luz Teresa Cañarte Quimis; José Luis Merino Murillo; Mariana de Lourdes Cantos Figuero

- La Jefa del Departamento de Talento Humano desconoce de la importancia en aplicar indicadores de gestión por tanto no se aplica medidas de desempeño para al personal en función de indicadores y de esta manera cumplir con los objetivos propuestos (Sánchez \& Lucio, 2017).

- La Jefa del Departamento de Talento Humano debe evaluar constantemente el desempeño laboral de los servidores públicos en los parámetros como la productividad, el clima laboral y el uso adecuado de los recursos (Sánchez \& Lucio, 2017).

- El GAD del Cantón Jipijapa no cuenta con un Manual de Clasificación de Puestos ya que existe desconocimiento del tema por parte de la administración, ocasionando a la institución un déficit en el desempeño del Talento Humano (Sánchez \& Lucio, 2017).

\section{Recomendaciones.}

- Es necesario que todo cuanto está plasmado en el modelo de gestión de talento humano se lo ponga en consideración y se aplique para poder dar una valoración a las diferentes áreas de trabajo, como también trabajar con planes de capacitación según sea la necesidad de cada área y realizar la evaluación de desempeño de las personas y así saber si están cumpliendo con lo previsto en cuanto a metas y objetivos trazados de forma anual. (Vallejos \& Robinzon, 2017).

- Se recomienda al Departamento de Talento Humano aplicar este método de

selección de personal, para que escojan profesionales de acuerdo al perfil que necesita la 


\section{Análisis de la cultura organizacional en los departamentos de talento humano}

y financiero del G.A.D de Jipijapa

Vol. 1, núm. 5., (2017)

Miguel Augusto Baque Cantos; Luz Teresa Cañarte Quimis; José Luis Merino Murillo; Mariana de Lourdes Cantos Figuero

institución y de esta manera cumpla con las expectativas para el cargo, mediante méritos y oposición (Sánchez \& Lucio, 2017).

- Al Alcalde y a la Jefa de Talento Humano tomar correcciones y aplicar las políticas y procedimientos para la contratación del personal, donde debe realizarse de una forma idónea (Sánchez \& Lucio, 2017).

- Se recomienda a la Jefa del Departamento de Talento Humano implementar el uso de los indicadores de gestión, que le permitan medir la eficiencia, eficacia y efectividad de los servicios y de esta manera proyecte una imagen institucional de calidad (Sánchez \& Lucio, 2017).

- La jefa de departamento de talento humano Debe evaluar utilizando los parámetros de productividad, clima laboral, etc.; y de esta manera conocer los avances de la gestión en el desempeño laboral organizacional de los procesos internos (Sánchez \& Lucio, 2017).

- Se recomienda a la Jefa del Departamento de Talento Humano y al Alcalde incorporar un Manual de Clasificación de Puestos, para que la institución designe las tareas correspondientes a través de análisis de competencia y méritos de oposición (Sánchez \& Lucio, 2017).

- Establecer un Plan Operativo Anual, de las acciones y recursos para un mejor desempeño administrativo acorde a todas las exigencia y de esta manera se logrará fortalecer 


\section{Análisis de la cultura organizacional en los departamentos de talento humano y financiero del G.A.D de Jipijapa}

Vol. 1, núm. 5., (2017)

Miguel Augusto Baque Cantos; Luz Teresa Cañarte Quimis; José Luis Merino Murillo; Mariana de Lourdes Cantos Figuero

el desarrollo institucional del Gobierno Autónomo Descentralizado del cantón Jipijapa (Villacreses, 2017).

- $\quad$ Es necesario que el G.A.D municipal del Cantón Jipijapa ejecute este modelo de procesos administrativos, que permitirá al Gobierno Autónomo Descentralizado Municipal del Cantón Jipijapa contar con una estructura que ayudara a mejorar la eficacia, eficiencia, planificación y evaluación de la institución (Villacreses, 2017).

\section{Bibliografía.}

Álvarez, D., \& Guachi, C. (2017). Análisis del Sistema de Control Interno y su Impacto en el Proceso de Ejecución Presupuestaria del Gobierno Autónomo Descentralizado del Cantón Jipijapa. Jipijapa: UNESUM.

Álvarez, D., \& Vera, M. (2017). La Gestión Administrativa y de Control y su incidencia en el Área de Gestión de Planificación y del territorio del Gobierno Autónomo descentralizado Municipal del Cantón Jipijapa. Jipijapa: UNESUM.

Fernández, L. (2017). Las políticas institucionales que norman los procesos en el departamento de talento humano del Gobierno Autónomo Descentralizado del Cantón Santa Ana. Jipijapa: UNESUM.

Gobierno Autonomo Descentralizado del Cantón Jipijapa. (2018). Jipijapa. GAD: GAP.

Mora, N. (07 de 03 de 2018). La Investigación Bibliográfica. Ideas principales y secundarias. Obtenido de http://fido.palermo.edu/servicios_dyc///////blog/docentes/trabajos/17306_55962.pdf

Sánchez, J., \& Lucio, A. (2017). Auditoria de Gestión al Talento Humano y su impacto en el proceso de nomina del GAD Cantonal Jipijapa del año 2015. Jipijapa: UNESUM.

Vallejos, C., \& Robinzon, F. (2017). Modelo de gestión de talento humano por competencias para el Consejo Cantonal de protección de derechos del cantón Antonio Ante. Ambato: UNIANDES.

Villacreses, C. (2017). Los procesos administrativos del gobierno autónomo descentralizados del cantón Jipijapa y su incidencia en el desarrollo institucional. Jipijapa: UNESUM. 